\title{
Technology Insight: proton beam radiotherapy for treatment in pediatric brain tumors
}

Torunn I Yock and Nancy J Tarbell

doi:10.1038/ncponc0163

\section{CORRIGENDUM}

In the December 2004 issue, Figure 3a in the Technology Insight review by Yock and Tarbell incorrectly depicts photons and intensitymodulated radiation therapy (IMRT) rather than protons and photons (X-rays). Protons have a sharp radiation dose reduction with no exit dose deposited to normal tissues beyond

A
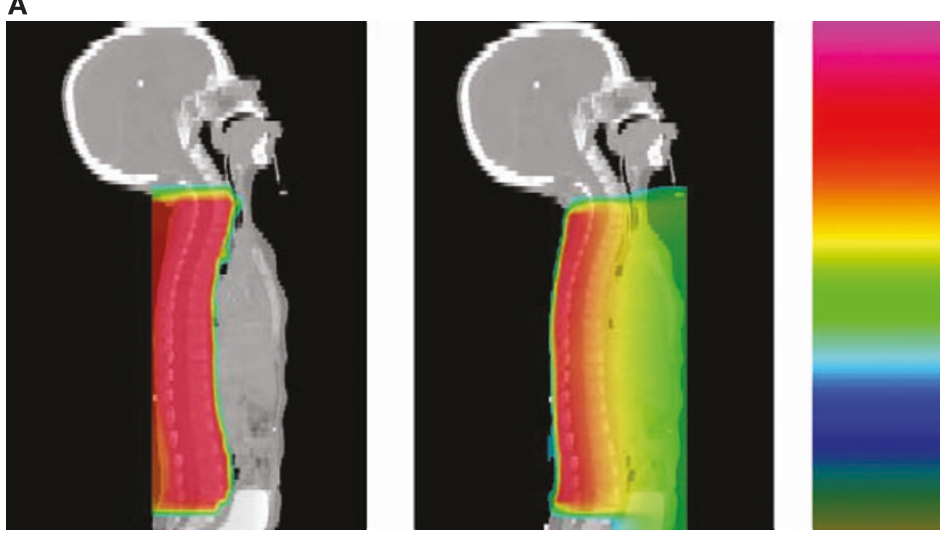

$120 \%$

Protons

X-rays
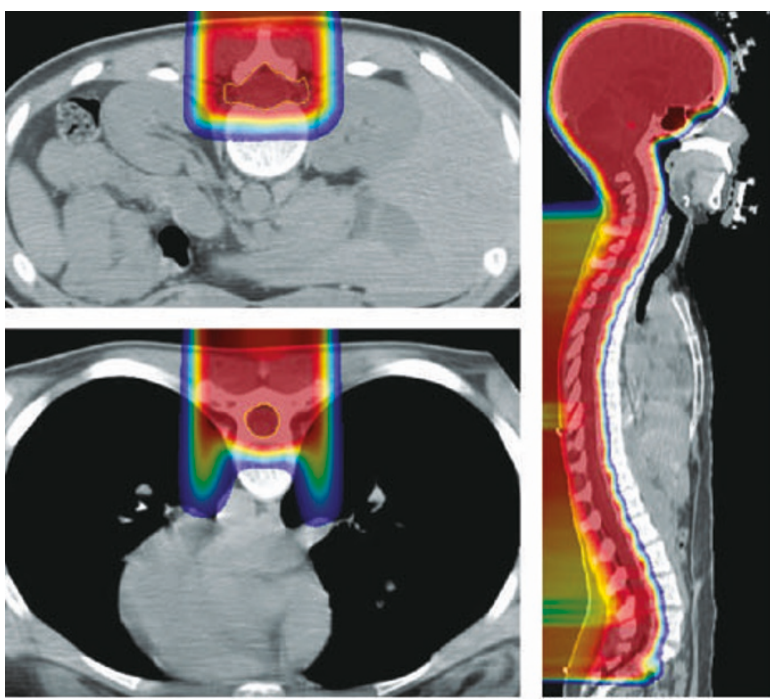

Dose (cGy)

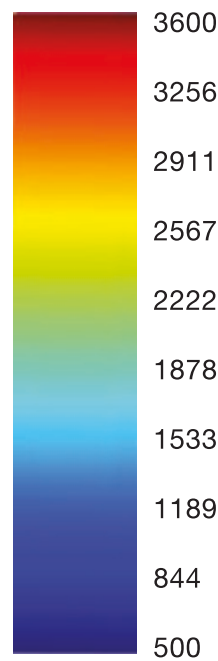

Figure 3 Differences in doses delivered to the spine and body using photons (X-rays) and protons.

(A) Radiation dose to the spine and anterior structures using protons and X-rays for comparison.

(B) Radiation dose to the vertebral body when only the thecal sac is treated in a patient who is fully grown. 